\title{
Simultaneous EELS/EDS Composition Mapping at Atomic Resolution Using Fast STEM Spectrum-Imaging
}

\author{
Paolo Longo, * Paul Thomas, Aziz Aitouchen, Bernhard Schaffer, and Ray D. Twesten \\ Gatan Inc., 5794 W Las Positas Blvd., Pleasanton, CA 94588
}

*plongo@gatan.com

\section{Introduction}

With advancements in aberration-corrected electron optics, the resolution in scanning transmission electron microscopy (STEM) has been significantly improved [1]. More importantly, the reduction of the probe size and the increase of the probe current density enable the acquisition of elemental maps at the atomic scale in a fast manner using both EELS and EDS. With the latest generations of EELS spectrometers [2], atomic resolution compositional and chemical maps allow interfaces [3], oxidation state, and even single atoms $[4,5]$ to be examined with increasing detail. These improvements have also enabled elemental and chemical maps to be acquired rapidly using both low- and high-energy edges from elements across the periodic table, including heavy atoms such Au or Pt [6-9]. On the EDS side, the introduction of large-area silicon drift detectors (SDD) $[10,11]$ has allowed these high-beam-current sources to be fully utilized. Improved detector area and support for higher count rates, compared to the previous generation of EDS detectors, allows the acquisition of EDS intensity maps from most types of material in the STEM, even for moderately thin samples. For the case of light elements, the reduced detector dead layer of SDD-based systems partially overcomes the very low florescence yield typical of low-energy X-ray lines. Also as reported in [11], atomic-level X-ray maps using fast detectors and bright sources can now be collected under some conditions. Because EDS and EELS provide complementary information about the sample, and are both generated with the electron beam, it would be wasteful to not acquire both datasets with every sample run.

Simultaneous EELS/EDS acquisition systems have been available since the late 1990s, but those systems are unable to keep up with the data rates of modern spectrometers. Recent advances in acquisition strategies and hardware have overcome the two main challenges of joint EELS/EDS data acquisition: (1) the exact pixel-to-pixel synchronization among different pieces of hardware, such as analog STEM detectors, serial counting EDS detectors, and parallel imaging EELS detectors, and (2) the vast amount of information that is generated by the system, from tens of kbits/s for the EDS pulses to the many MBytes/s of data from the EELS detector. As described in [6], these challenges have been overcome by acquiring the data in a hardware-synchronized mode. The individual acquisition systems collect their data independent of the other but are linked together by a timing signal. The synchronization signal is delivered by the EELS spectrometer by means of timing pulse at the end of each acquired EELS spectrum. This pulse is used to advance the beam position and inform the EDS system that the pixel has advanced, resulting in a highly efficient detection system with very little pixel-topixel dead time. Following initial successful results of simultaneous high-speed EELS and EDS analysis from a catalyst sample containing PdAu nanoparticles [6], in this article we use this acquisition method to create the first reported high-speed jointly acquired EELS and EDS spectrum images at atomic resolution.

\section{Methods and Materials}

Instrumentation. Data was acquired at TU-Graz in Austria using a probe-corrected FEI Titan G2 STEM equipped with a X-FEG high-brightness electron source operating at $200 \mathrm{kV}$. The instrument is also equipped with a four-quadrant SDD detector (FEI Super-X) [10], controlled by the Bruker Esprit system and a GIF Quantum ERS imaging filter for EELS acquisition [2]. The EELS spectrometer is configured with the following features: (a) DualEELS ${ }^{\mathrm{TM}}$ capability, which allows two different regions of the EELS spectrum to be recorded nearly simultaneously $(\Delta t=10 \mu \mathrm{s})[2,12]$; (b) a low dispersion mode, which allows EELS data to be recorded with an energy range up to $2000 \mathrm{eV}$ (or $4000 \mathrm{eV}$ in DualEELS mode); (c) a fast 2-k CCD, which allows EELS acquisition at over 1000 spectra per second [2]; and (d) a dodecapole lens system capable of correcting spectral aberrations up to the 5th order, allowing collections angles well over $100 \mathrm{mrad}$ to be used while maintaining sub-eV energy resolution [2].

Test specimen. The sample analyzed was a $\mathrm{SrTiO}_{3}(100)$ crystal. A relatively thick sample for this type of measurement ( $>0.7 \mathrm{t} / \lambda$ as measured by low-loss EELS) was used to generate sufficient EDS counts for the analysis. This results in some loss of spatial resolution.

Acquisition parameters. Data were acquired using a STEM probe of $160 \mathrm{pA}$ measured on the microscope fluorescence screen. This STEM microscope is capable of much higher current, but a relatively low current was used in order to reduce the beam damage. The probe convergence angle was $22 \mathrm{mrad}$, and the EELS collection angle was estimated to be just over $40 \mathrm{mrad}$. The EDS collection angle is reported to be $\sim 0.7$ sr but was not independently measured in these experiments. Three different signals (ADF, EDS, and EELS core-loss) were recorded simultaneously. The region of the EELS spectrum from $300 \mathrm{eV}$ to $2300 \mathrm{eV}$ was acquired using a dwell time per pixel of $10 \mathrm{~ms}$. The EDS spectrum was acquired simultaneously, integrating counts over the entire pixel dwell time employed for the EELS acquisition. The entire $70 \times 57$ pixel spectrum imaging dataset was taken in just 48 seconds. This is the time from the start to the moment when all the EDS and EELS spectra were transferred from the hardware buffers to the Gatan computer. The actual acquisition time was somewhat shorter ( $\sim 40$ seconds) but was not directly measured. It is important to mention that no drift correction was employed during the acquisition of the spectrum imaging (SI). Because the sample was inserted into the microscope less than 1 hour before the data were collected, some residual spatial drift was observed in the elemental maps. 


\section{Moving on up ...}

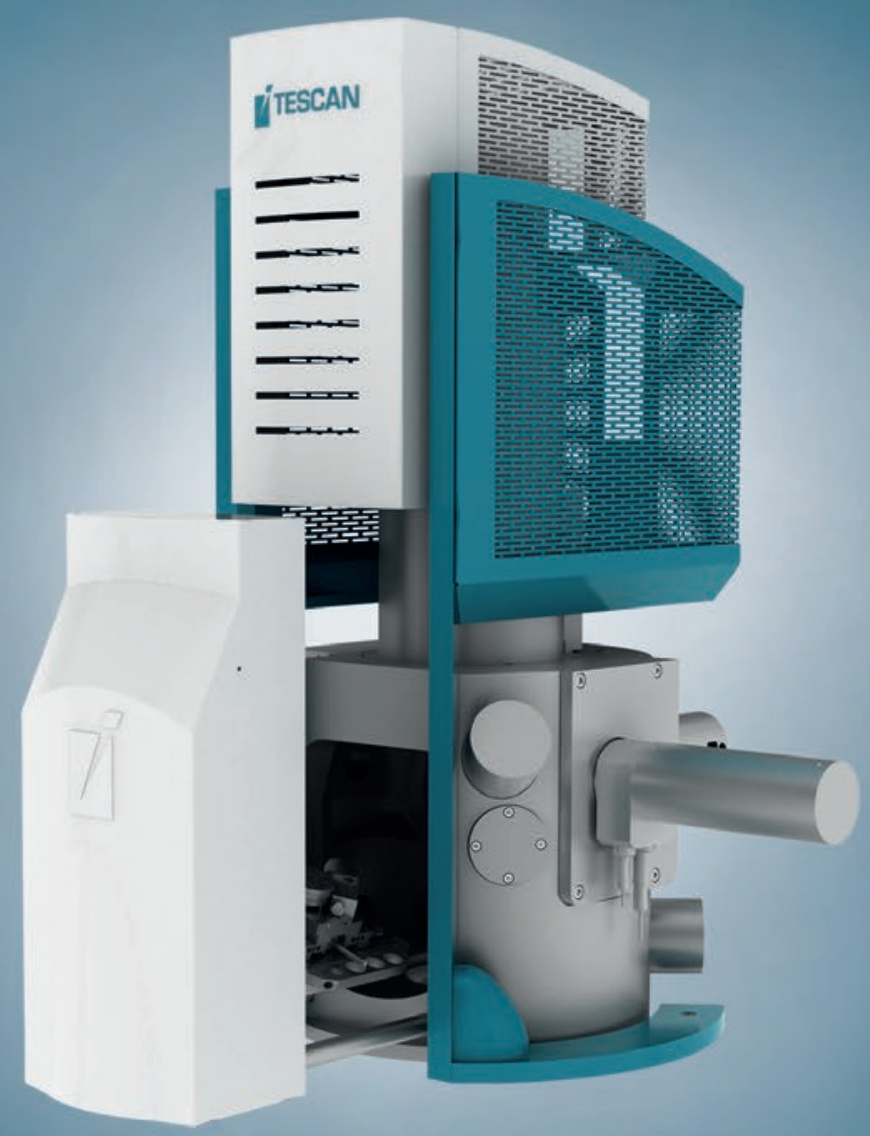

Reliability, performance, superior analytical flexibility and ease of use are just a few reasons the TESCAN VEGA thermionic scanning electron microscope is fast becoming the number one choice among electron microscopists.

\section{Come visit us at booth $\mathbf{5 2 8}$ in Indianapolis and find out why more and more researchers are choosing TESCAN.}

*Thermionic Scanning Electron Microscopes

*Field Emission Scanning Electron Microscopes

*FIB-SEM Workstations

*FIB-SEM Time of Flight Integration

*Plasma FIB-SEM Workstations

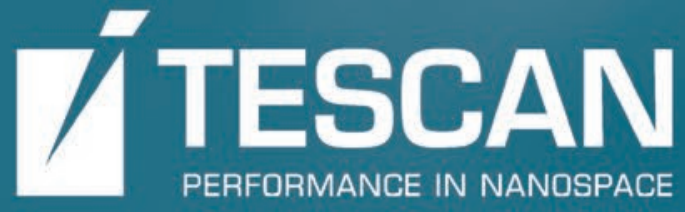

www.tescan.com www.tescan-usa.com 


\section{Results}

Figure 1 shows the ADF image used as a survey image for the acquisition of the EELS and EDS SI. EELS and EDS elemental maps were taken from the area highlighted in the green box. Figures $2 b$ and $2 c$ show EELS and EDS spectra from a single pixel extracted from the region in the red box shown in the EELS SI in Figure 2a. The EELS spectrum shows a reasonable amount of signal. The $\mathrm{Sr} \mathrm{L}_{2,3}$-edges at $1940 \mathrm{eV}$ stand out quite well above the background, and the entire EELS spectrum contains approximately $665 \mathrm{k}$ electrons. Unlike EELS, the EDS spectrum shows a limited number of counts, and the Sr L and Ti $\mathrm{K}$ lines are barely detectable. There are approximately $117 \mathrm{X}$-ray counts in the entire spectrum that extends up to $20 \mathrm{keV}$. The stronger signal in the EELS spectrum results in reasonable signal-to-noise ratio (SNR) at the individual spectrum level even with this short exposure time $(10 \mathrm{~ms} / \mathrm{pixel})$. The small number of counts in the EDS spectrum precludes any quantification at the single-spectrum level. EELS elemental maps of $\mathrm{Sr} \mathrm{L}_{2,3}$-edges at $1940 \mathrm{eV}$, Ti $\mathrm{L}_{2,3}$-edges at $456 \mathrm{eV}$, and $\mathrm{O}$ K-edge at $532 \mathrm{eV}$ were extracted using MLLS fitting $[13,14]$ as previously described in [7]. In the case of EDS, elemental maps were extracted using the $\mathrm{Sr} \mathrm{L}_{\mathrm{a}}$ lines at $1.81 \mathrm{keV}$ and the $\mathrm{Ti} \mathrm{K}_{\mathrm{a}}$ at $4.51 \mathrm{keV}$ using an empirical Kramers background [15] fitting followed by multiple linear least square (MLLS) fitting of each peak family represented by a set of Gaussian peaks. $\mathrm{X}$-ray maps using the $\mathrm{O} \mathrm{K}$ line were attempted, but because of the limited signal and also the overlap of the $\mathrm{O} K$ and $\mathrm{Ti} \mathrm{L}$ peaks, the results were unsatisfactory.

Figures $3 \mathrm{a}$ and $3 \mathrm{~b}$ show the Ti elemental maps obtained using EELS and EDS, respectively. Qualitatively, there is less noise and higher contrast in the EELS map compared to that from EDS. Figures $4 \mathrm{a}$ and $4 \mathrm{~b}$ show the Sr elemental maps obtained using EELS and EDS, respectively. In both cases the Sr L transition was used. As in the case of the Ti elemental maps, the EELS map shows less noise and seems sharper than that obtained using EDS. To measure the relative quality of each map, the signal intensity was analyzed from a uniform region across each single elemental map. This $5 \times 5$ pixel region is indicated by the red boxes in Figures 3 and 4 . The extracted data from the $5 \times 5$ pixel region are summarized in Table 1 . In the case of the Ti elemental maps, the SNR for the EELS data

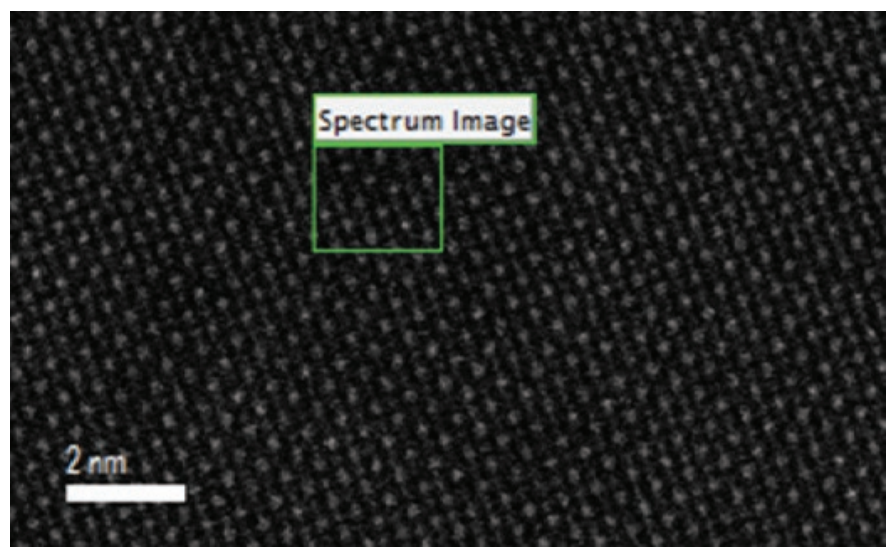

Figure 1: An ADF STEM survey image acquired using the STEM detector fitted above the fluorescence screen. The green box is the area where the simultaneous EELS and EDS SI were taken. was $\sim 30$, almost 7 times greater than the EDS data. In the case of the $\mathrm{Sr}$ elemental maps, the SNR was $\sim 11$ and $\sim 5$ for EELS and EDS, respectively, giving over $2 \times$ improvement for the EELS data. The SNR in the EDS data is limited by Poisson statistics, and the collection efficiency would need to be more greatly improved ( 2 times for the Sr and over 6 times for the Ti) even
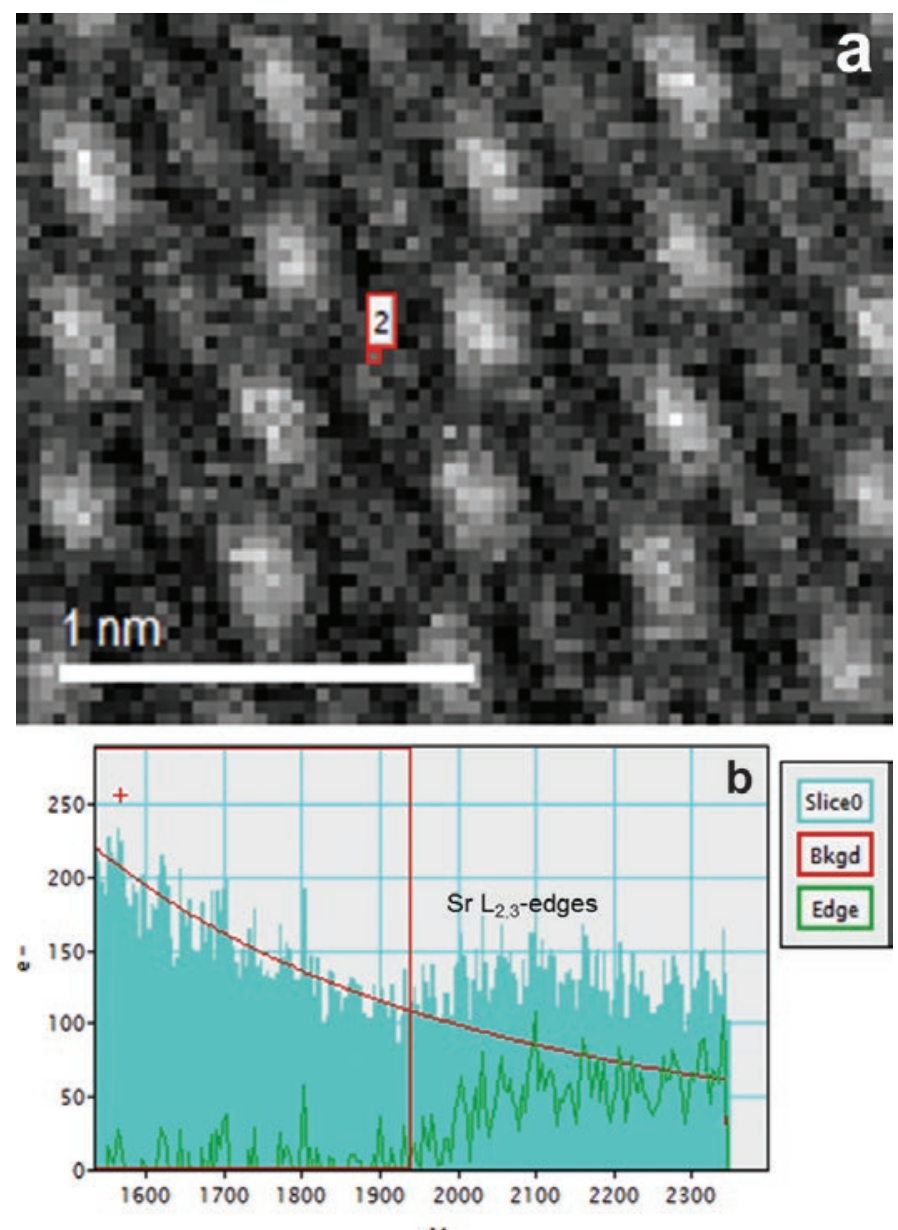

eV

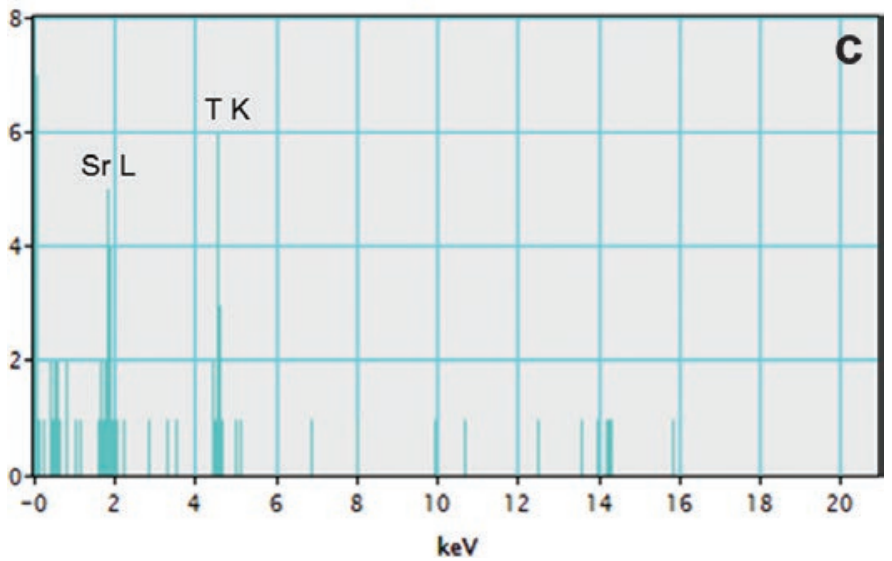

Figure 2: a) An energy-filtered STEM image (energy range 1.4-2.3 keV) extracted from the EELS SI dataset. Each point in the EELS SI dataset is a complete EELS spectrum. A corresponding EDS SI dataset is co-acquired with the EELS data. A single b) EELS spectrum and c) EDS spectrum extracted from the point marked in a) shows the complementary nature of the multi-modal data acquisition. EELS data show high signal intensity even at the single pixel level. EDS data show weak intensity at the single pixel level, but the low background allows pixel averaging to increase signal at reduced spatial resolution. 

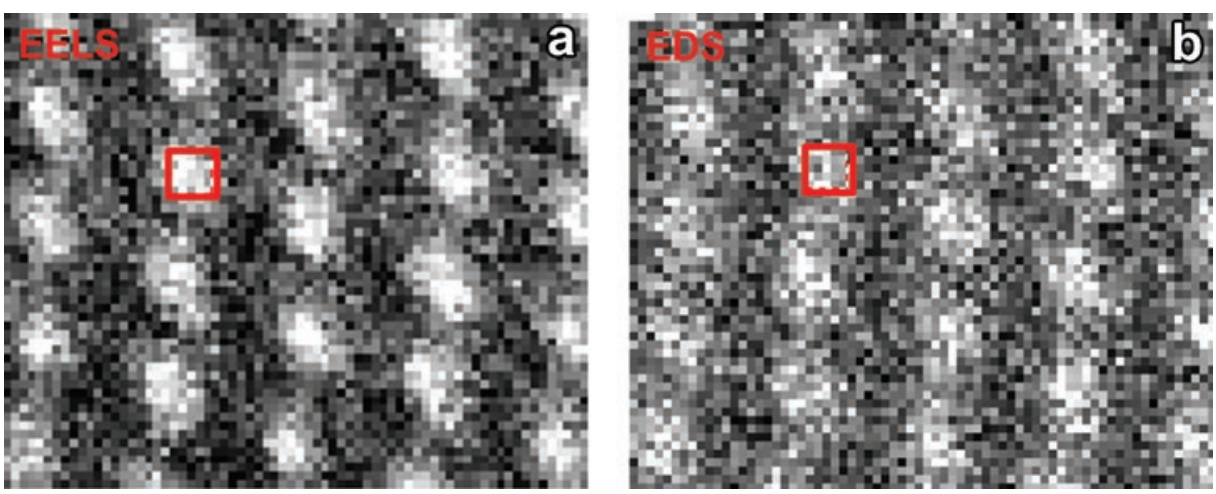

Figure 3: Ti Elemental maps: a) EELS using the Ti $\mathrm{L}_{2,3}$-edges at $456 \mathrm{eV}$. b) EDS using the Ti $\mathrm{K}_{\alpha}$ at $4.51 \mathrm{keV}$. The area in the red box is where the SNR measurements reported in Table 1 were made.
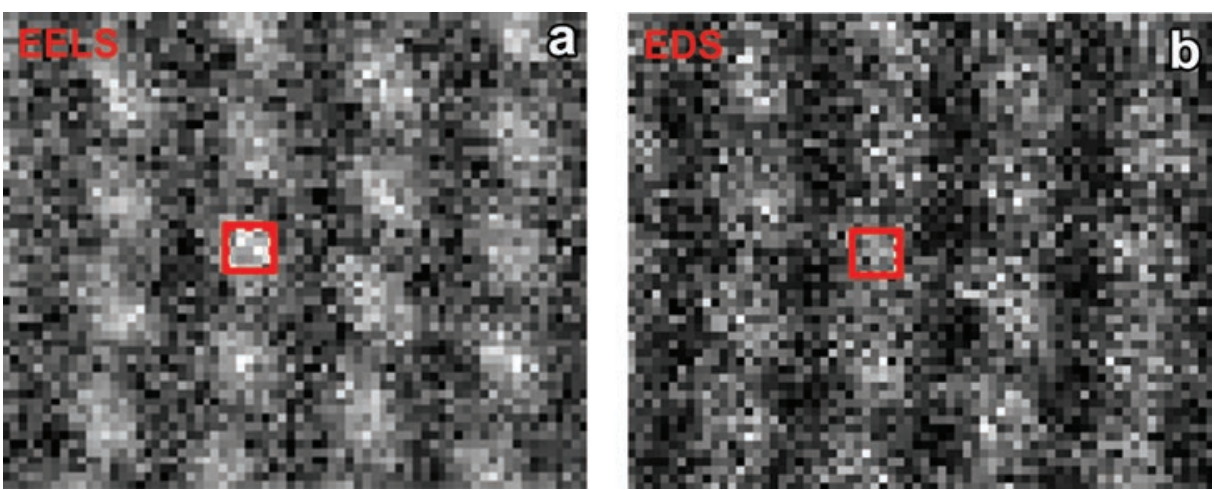

Figure 4: Sr Elemental maps: a) EELS using the $\mathrm{Sr} \mathrm{L}_{2,3}$-edges at $1940 \mathrm{eV}$. b) EDS using the $\mathrm{Sr} \mathrm{L}_{\alpha}$ at $1.81 \mathrm{keV}$. The area in the red box is where the SNR measurements reported in Table 1 were made.
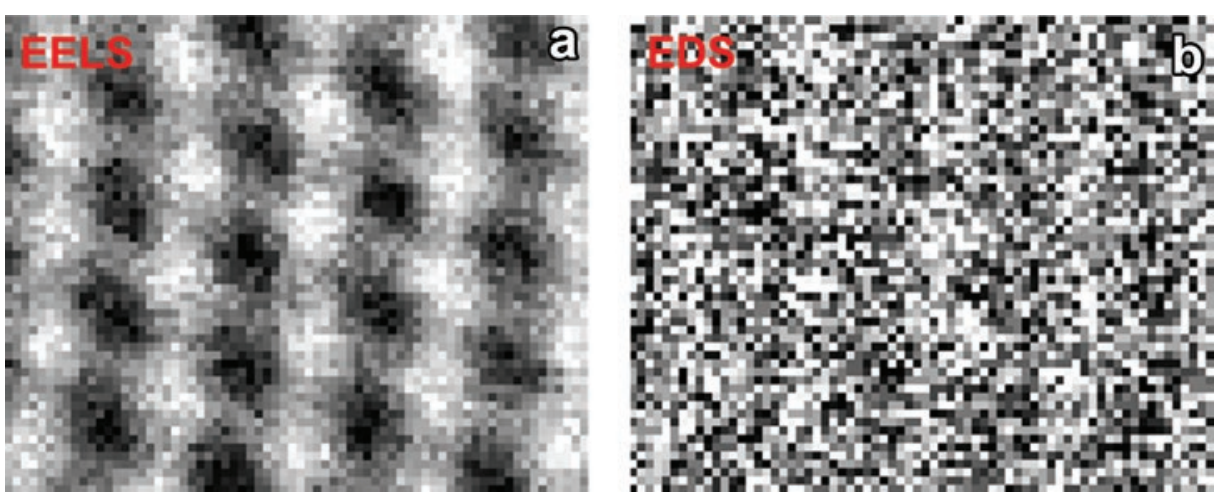

Figure 5: O elemental maps: a) EELS using the O K-edge at $532 \mathrm{eV}$. b) EDS using the $\mathrm{K}_{\mathrm{a}}$ at $0.52 \mathrm{keV}$.

As mentioned above, the EDS data show no oxygen structure.

Figures $6 \mathrm{a}$ and $6 \mathrm{~b}$ show colorized elemental maps of $\mathrm{Ti}$ and $\mathrm{Sr}$ obtained using EDS and EELS, respectively. The left half of each color map shows the raw experimental data, whereas the right half shows the data after smoothing out of some of the noise. Qualitatively, the EELS maps appear to be sharper and show stronger contrast. The distinction between EELS and EDS elemental maps is more clearly seen in the raw data, emphasizing the superiority of EELS at the single-spectrum limit and the misleading effects of strong spatial averaging as a result of the smoothing procedure employed to de-noise the data.

\section{Discussion}

The EELS and EDS data presented here can be regarded as highly complementary. The main difference is that for the EDS case, each individual pixel carries little information because of the low SNR of the data. However, the high peak-to-background ratio of the EDS technique allows summation of adjacent data, almost indefinitely improving the detection limits at the expense of spatial resolution. The EELS data, on the other hand, has very high collection efficiency resulting in high SNRs in each individual spectrum. Summing adjacent pixels improves the detection limits considerably but will show diminishing returns once the shot noise in the signal integral becomes irrelevant. The techniques further complement each other when identifying artifacts in the data. For example, secondary fluorescence and preferential absorption in the EDS data can be determined from comparison with the EELS data, and possible mis-

Table 1: SNR measurements.

\begin{tabular}{|l|c|c|c|}
\hline & Mean Signal & Std. Dev. & SNR \\
\hline Ti L EELS Map & 81 a.u. & 2.8 a.u. & 28.9 \\
\hline Ti K EDS Map & 24.3 a.u. & 5.5 a.u. & 4.4 \\
\hline Sr L EELS Map & 88.8 a.u. & 7.9 a.u. & 11.2 \\
\hline Sr L EDS Map & 25.5 a.u. & 4.9 a.u. & 5.2 \\
\hline
\end{tabular}

for the relatively thick samples used here. Figures $5 \mathrm{a}$ and $5 \mathrm{~b}$ show the O K elemental maps using EELS and EDS, respectively. The EELS data does not completely resolve individual $\mathrm{O}$ atoms, but it does show the distributed oxygen framework. sing elements in the EELS data range acquired can be identified in the summed EDS signal. Furthermore, the ability of combining composition measurements from EELS and EDS with physical and electronic information also available only from EELS (for example, plasmonic, bandgap, and density of states information) [16] promises to open whole new avenues of materials analysis.

\section{Conclusions}

A system for fast and efficient acquisition of both EELS and EDS spectra allows the routine collection of both of these complementary data signals. There is no longer a need to choose between analytical techniques; both EELS and EDS can be acquired easily with every data run. The data presented here 

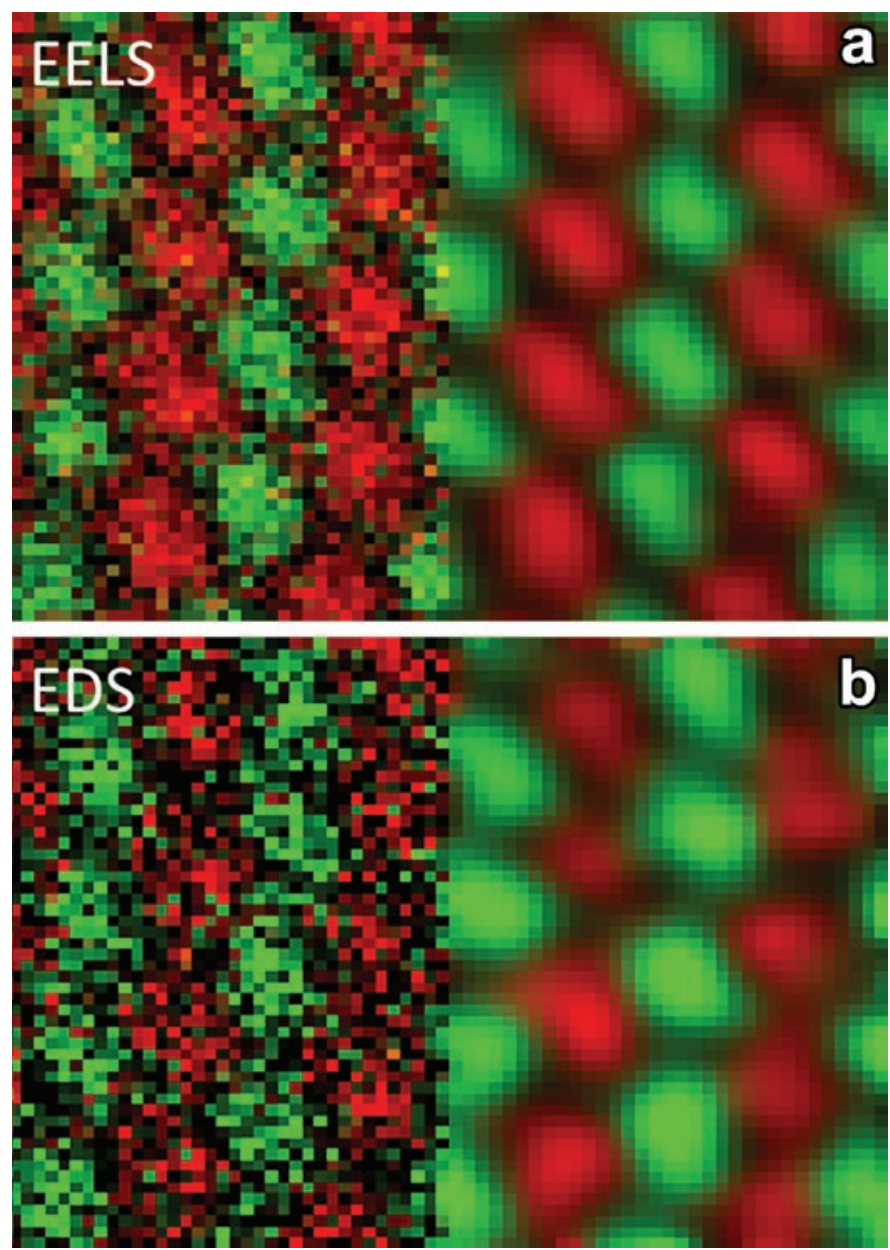

Figure 6: Colorized elemental maps of $\mathrm{Sr}$ in red and $\mathrm{Ti}$ in green acquired using EELS and EDS. The left half parts are the raw experimental maps, whereas the right half shows filtered maps.

show the complementary nature of having both high signalto-noise and high peak-to-background data acquired at the same location at the same time.

\section{Acknowledgments}

We would like to thank Dr. Yan Xin of Florida State University for kindly providing the TEM specimen used for the experiment, Professor Gerald Kothleitner of TU-Graz for many discussions and access to the TEM facilities, and Mr. Arno Meingast for expert assistance configuring the microscope for this experiment.

\section{References}

[1] DA Muller, L Fitting Koukoutis, M Murfitt, JH Song, HY Hwang, J Silcox, N Dellby, and OL Krivanek, Science 319 (2008) 1073-76.

[2] AJ Gubbens, M Barfels, C Trevor, RD Twesten, PJ Thomas, N Menon, B Kraus, C Mao, and B McGinn, Ultramicroscopy 110 (2010) 962-70.

[3] EJ Monkman, C Adamo, JA Mundy, DE Shai, JW Harter, D Shen, B Brganov, DA Muller, DG Schlom, and KM Shen, Nature Materials 11 (2012) 855-59.

[4] H Tan, S Turner, E Yucelen, J Verbeeck, and G Van Tendeloo, Phys Rev Lett 107 (2011) 107602.
[5] K Suenaga and M Koshino, Nature 468 (2010) 1088-90.

[6] P Longo and RD Twesten, Microscopy Today 21(1) (2013) 28-33.

[7] P Longo, RD Twesten, and PJ Thomas, Microscopy Today 20(4) (2012) 30-36.

[8] P Longo, Gatan Inc., Applications Note 2012: http://www .gatan.com/files/PDF/products/app_notes/AppNote_ High_Speed_EELS_Longo_FL1.pdf

[9] P Longo, Gatan Inc., Applications Note 2011: http://www .gatan.com/files/PDF/st_simul_acquisition_EELS_FL .pdf

[10] P Schlossmacher, DO Klenov, B Freitag, and S Von Harrach, Microscopy Today 18(4) (2010) 14-20.

[11] LJ Allen, AJ D'Alfonso, B Freitag, and DO Klenov, MRS Bulletin 37 (2012) 47-52.

[12] J Scott, PJ Thomas, M Mackenzie, S McFadzean, AJ Craven, and WAP Nicholson, Ultramicroscopy 108 (2008) 1586-94.

[13] P Thomas, Gatan Inc., Knowhow, Dec. 14, 2006: http:// www.gatan.com/resources/knowhow/kh14-spectral .php

[14] K Riegler and G Kothleitner, Ultramicroscopy 110 (2010) 1004-13.

[15] E Lifshin, Proceedings of the 9th Ann. Conference of the Microbeam Analysis Society, Ottawa, Canada (1974) 53.

[16] RF Egerton, Electron Energy-Loss Spectroscopy in the Electron Microscope, Singer, New York, 1996.

\section{PELCO silicon Nitride \& Silicon Dioxide Membranes}

\section{Next Generation SiN TEM Support Films}

- Robust and clean 8, 15, 50 and $200 \mathrm{~nm}$ SiN substrates

- $ø 3.0 \mathrm{~mm}$ frame

- EasyGrip ${ }^{\text {Th }}$ edges

- Free from debris

- Super flat 8, 15, and 40nm silicon dioxide substrates
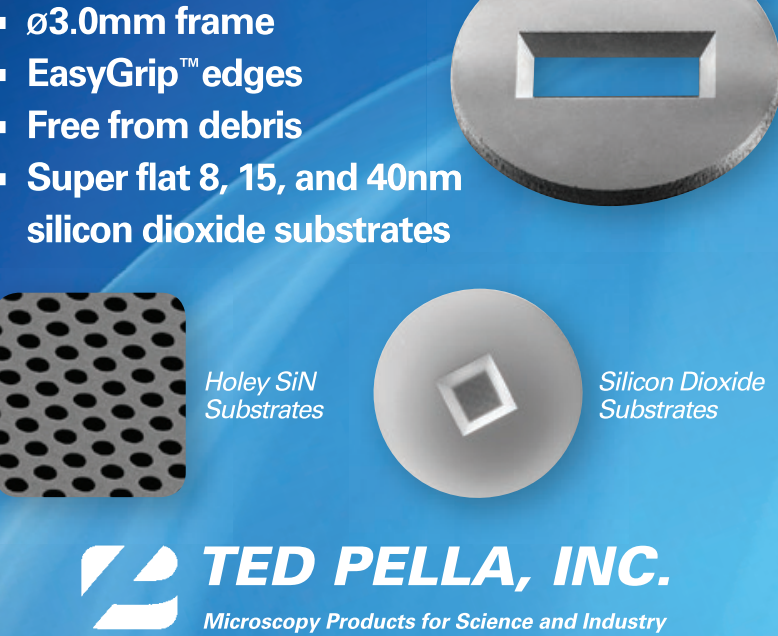

www.tedpella.com sales@tedpella.com 800.237.3526 

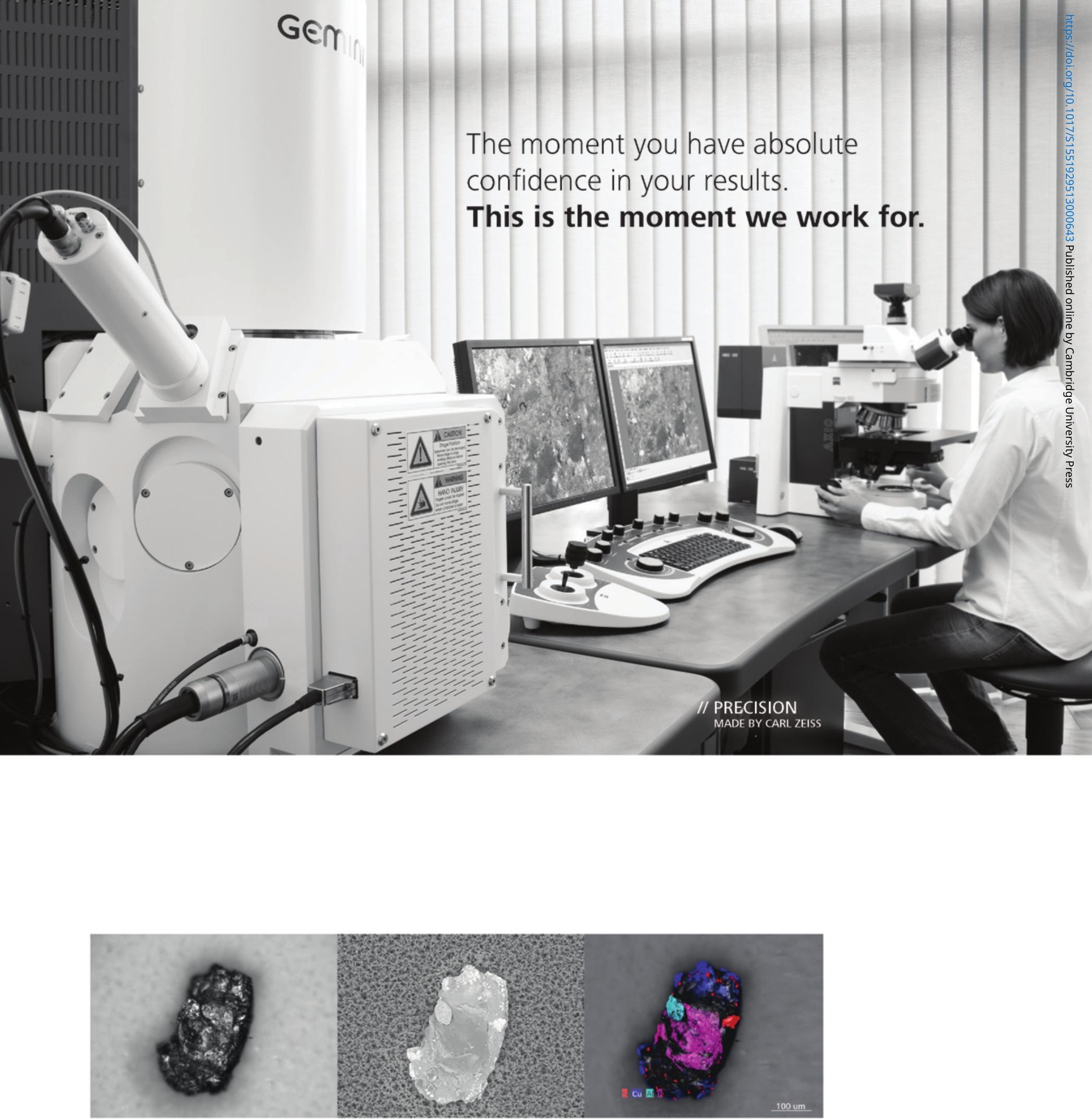

Metal particle imaged with light microscopy (left), electron microscopy (middle) and precise overlay for correlative analysis with elemental composition from EDX (right)

Correlative microscopy enables you to discover more information from your sample by precisely overlapping data from both light and electron microscopy. Only Carl Zeiss manufactures both light and electron microscopes for seamless integration. Contact us to request a brochure, quote or appointment with your local account manager.

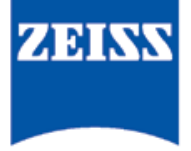

We make it visible. 